\title{
COMPARISON AND CORRELATION OF NANOFILLER AND NANOHYBRID COLOR STABILITIES WITH BARIUM LEACHING
}

\author{
Muchlis Fauzi Nasution', Felicia Maria' ${ }^{1}$ Endang Suprastiwi², Nilakesuma Djauharie², Aditya Wisnu Putranto² \\ 'Residency Program, Department of Conservative Dentistry, Faculty of Dentistry, Universitas Indonesia, Jakarta, Indonesia \\ ${ }^{2}$ Department of Conservative Dentistry, Faculty of Dentistry, Universitas Indonesia, Jakarta, Indonesia
}

\begin{abstract}
INTRODUCTION: Currently, there are two latest composite resin materials: nanofiller and nanohybrid composite resins. Additional compositions in nanofiller and nanohybrid composite resins are expected to significantly impact color stability. Color stability in a composite resin can be influenced by two factors, such as intrinsic and extrinsic elements, which also influence each other. Camphorquinone is the main cause of internal staining in composite resins, whereas tannins and curcumin are the highest causes of external staining in composite resins to date. Color stability can be evaluated using several tests, including color change test, filler leaching test, water absorption, resin solubility test, and surface roughness test.

ОвJеCTIVEs: This study was conducted to analyze a correlation of barium leaching with color stability of nanofiller and nanohybrid composite resins.

MATERIAL AND METHODS: A total of 20 specimens divided into 4 groups: group 1 (nanofiller) and group 2 (nanohybrid) composite resins (control groups) immersed in artificial saliva, and group 3 (nanofiller) and group 4 (nanohybrid) composite resins immersed in turmeric solution and coffee solution, and subjected to tooth brushing treatment, were analyzed in this study. Each group of composite resins was subjected to FESEM/EDX (field emission scanning electron microscopy with energy-dispersive X-ray) and colorimeter evaluation.

RESULTS: A correlation between color stability and barium leaching was observed, with a significant conversion strength of $64.7 \%$ (strong correlation) $(p<0.05)$. A correlation between color stability and barium leaching was observed, with a significant conversion strength of $64.7 \%$ (strong correlation) $(p<0.05)$.

Conclusion: Color stability of nanofiller composite resins was better than that of nanohybrid composite resins.
\end{abstract}

KEY WORDS: tnanotechnology, barium, composite resin.

J Stoma 2020; 73, 6: 295-301

DOI: https://doi.org/10.5114/jos.2020.101874

\section{INTRODUCTION}

A composite resin consists of a matrix, a filler, a coupling agent, a photoinitiator, and other components. The advancement in nanotechnology in the field of dentistry have resulted in a creation of composite resins con- taining nanosized particles. Currently, there are two types of composite resins based on nanotechnology, i.e., nanofiller and nanohybrid [1-4].

Nanohybrid composite resin consist of combined fillers, ranging from nanophils to modified macrophils, which are pre-polymerized with the resin matrix from

\section{JOURNAL OF} STOMATOLOGY

AdDress For CORRESPONDENCE: Prof. Endang Suprastiwi,

Department of Conservative Dentistry, Faculty of Dentistry, Universitas

Indonesia, Jakarta, Indonesia, e-mail: esuprastiwi@yahoo.co.id

RECEIVED: 09.04.2020 • ACCEPTED: 21.07.2020 • PUBLISHED: 30.12.2020 
methacrylate group. Dispersants and camphorquinone are used as their coupling agents and photoinitiators [3].

A nanofiller composite resin uses nanomeric and nanocluster fillers, which are covalently bonded to resemble dense grapes. Resin matrixes are mixed with bisGMA, TEGDMA, bis-EMA, UDMA, and a coupling agent, such as photoinitiator, silane, and camphorquinone (CQ) [4].

The color stability of composite resins is influenced by intrinsic and extrinsic factors. Intrinsic factors include the type of matrix, size, composition and properties of fillers, coupling agent, and photoinitiator [5]. Extrinsic factors include foods and beverages that contain dyes, such as curcumin and tannin as well as cigarettes [6-9]. In addition to extrinsic coloring agents, several studies have described that due to the brushing process, the teeth can indirectly develop a composite resin surface roughness, which can trigger color changes. However, other studies have reported that a tooth brushing process can actually help removing extrinsic stains, which physically bind to the surface of the teeth as well as aid in composite resin restorations [10].

Previous studies have explained that color changes occurring during composite resin restoration have a correlation with water absorption [11]. In addition, water absorption is related to filler leaching [12-14]. The intended types of fillers used in this context are barium and strontium. Barium is selected due to its very small diameter, its continuous presence in a composite resin, and its nature, i.e., being reactive to water molecules, so that it can easily escape when hydrolysis occurs [14-16]. To our knowledge, there is no study till date that has discussed color changes in a composite resin, which have a direct relationship with filler leaching.

\section{OBJECTIVES}

This aim of this study was to compare and correlate color stability of nanohybrid and nanofiller composite resins using a color change test and a release of barium filler.

\section{MATERIAL AND METHODS}

This was a laboratory experimental study conducted between October and November 2018 at the Dental Materials Laboratory, Faculty of Dentistry, and Faculty of Metallurgical Engineering Laboratory, Universitas Indonesia, Indonesia.

Dependent variables included color change, release of barium filler, and water absorption. Controlled variables were composite resins, i.e., a nanofiller (Filtek ${ }^{\mathrm{TM}}$ Z350XT 3M ESPE, St. Paul, MN, USA, 2010) and a nanohybrid (ceram. $\mathrm{x}^{\circledast}$ SphereTEC ${ }^{\mathrm{TM}}$ one universal, Dentsply Sirona, Charlotte, USA, 2015). The specimens were divided into samples for discoloration, with the number of bar- ium leaching of the same 20 specimens, and divided into 4 groups (6 mm wide and 2 mm thick, ISO 4049: 2000) with 1-year clinical exposure simulation (3 days of immersion and 10,950 cycles of tooth brushing). Before immersing the specimens in various solutions, more than 20 composite resin specimens were analyzed for the number of initial barium fillers using EDX/FESEM with the same pick up location. The middle area of the specimen was measured from the edge of specimen as many as 4 points. Then, 20 composite resin specimens were analyzed for the initial color using a $3 \times$ exposure colorimeter [17-20].

After measuring the initial color, each group was subjected to treatments as follows - Control group 1 (nanofiller): 5 nanofiller composite resin specimens were immersed in artificial saliva $(\mathrm{NaCl}, 0.7 \mathrm{~g}$; KSCN, $0.33 \mathrm{~g}$; $\mathrm{NaHCO}_{3}, 1.5$ g; urea, 0.13 g; $\mathrm{Na}_{2} \mathrm{HPO}_{4}, 0.26$ g; $\mathrm{KH}_{2} \mathrm{PO}_{4}$, $0.2 \mathrm{~g}$ ) for 6 days (color change analysis was conducted on days 3 and 6); Control group 2 (nanohybrid): nanohybrid composite resins were immersed in artificial saliva for 6 days (analysis of color changes was performed on days 3 and 6); Group 3: nanofiller composite resins were immersed in a turmeric solution (color analysis was done after 3 days), followed by immersion in a coffee solution (color analysis was conducted after 3 days), and then tooth brushing treatment (color analysis was performed after 10,950 cycles); Group 4: nanohybrid composite resins were immersed in a turmeric solution (color analysis was done after 3 days), followed by immersion in a coffee solution (color analysis was conducted after 3 days), and then tooth brushing treatment (color analysis was done after 10,950 cycles) $[9,10]$.

The color changes $(\Delta \mathrm{E})$ were calculated from the $\mathrm{L}^{*}$, $\mathrm{a}^{*}$, and $\mathrm{b}^{*}$ values for each specimen, according to the following formula, which determines the three-dimensional color space: $\Delta \mathrm{Elab}^{*}=\left[\left(\Delta \mathrm{L}^{*}\right)^{2}+\left(\Delta \mathrm{a}^{*}\right)^{2}+\left(\Delta \mathrm{b}^{*}\right)^{2}\right]^{1 / 2}$. After conducting the final color analysis, 20 specimens were analyzed using EDX/FESEM to determine the amount of barium leaching $[9,10,17,18,21]$.

Color change data of the composite resins were analyzed using parametric statistical tests in the SPSS 22 software. Statistical analyses were conducted with data normality and homogeneity tests. For non-homogeneous and normally distributed data, one-way ANOVA test was performed. Furthermore, a post-hoc Tamhane test was performed $(p<0.05)$ to assess the level of significance between the groups. Data related to barium leaching were also analyzed using a parametric statistical tests of data normality and homogeneity in the SPSS 22 software.

One-way ANOVA test was performed to analyze normally distributed and homogeneous data. In addition, the homogeneous data were subjected to Bonferroni post-hoc test $(p<0.05)$ to verify the significance between the groups. To analyze the presence of any relationship between discoloration and barium leaching, a correlation and simple linear regression tests were performed $(p<0.005)$ 


\section{RESULTS}

Color changes were evaluated by the post-hoc Tamhane test, and barium leaching data were assessed by the Bonferroni test $(p<0.05)$. To determine the correlation between barium leaching and color changes, a simple linear correlation test was conducted.

As shown in Table 1, the mean color change values were 0.56 (excellent) in group 1 and 1.45 (good) in group 2. In groups 3 and 4 , the mean color change values were 5.69 and 6.88 (mismatch), respectively. The values (evaluated by the post-hoc Tamhane test) among all the groups were significantly different $(p<0.05)$, indicating that the ability to change color in the nanohybrid composite resin group was higher than that in the nanofiller group, in cases of both untreated and treated resins.

As shown in Table 2, the mean values for barium leaching were higher in groups 4 and 2 nanohybrid composite resins. This results indicated that nanohybrid composite resins generated higher barium leaching than nanofiller composite resins. The values (evaluated by the post-hoc Bonferroni test) of barium leaching (\%) were significant among the nanohybrid composite resin group with treatment (group 4) and the group of untreated nanofiller composite resin (group 1), the group of untreated nanohybrid composite resin (group 2) and the group of nanofiller composite resin with treatment (group 3). Therefore, group 4 exhibited significant barium leaching.

The linear regression relationship resulted in a value of $64.7 \%$, which implied a strong and significant relationship between color changes and barium leaching $(p<0.05)$.

\section{DISCUSSION}

Color stability of composite resins can be analyzed with several tests, including color changes and barium leaching tests. To assess the occurrence of discoloration that simulates events in the oral cavity, several studies have suggested that more than one dye agent should be used separately. In this study, the exposure treatment was established as turmeric, coffee, and brushing with toothpaste [1-4, 6-10].

The process of water absorption by a composite resin triggers hydrolysis and release of fillers, which cause voids, so that the coloring agent easily attaches to the composite resin. According to Roeder et al., changes in color with a value of $<1 \Delta \mathrm{E}^{\star}$ cannot be evaluated visually, due to which colorimeters or spectrophotometers were used to detect up to a depth of several microns in this study. The limit of ${ }^{\star} \Delta \mathrm{E}^{\star}$ value in the composite resin should not be $>3.5[22,23]$. The results of this study demonstrate that there were more color changes in the nanohybrid composite resin than in the nanofiller composite resin in both the control and treatment groups.

In this study, color change values of 5.69 in group 3 (nanofiller) and 6.88 in group 4 (nanohybrid) were ob-
TABLE 1. Mean values and significance $(p)$ of color changes $\left(\triangle E^{*}\right)$ in groups $1-4$

\begin{tabular}{|l|l|c|c|}
\hline Group & $\boldsymbol{n}$ & Mean (SD deviation) & $\boldsymbol{p}$-value \\
\hline 1 & 5 & $0.56(0.15)$ & \multirow{2}{*}{$<0.000$} \\
\hline 2 & 5 & $1.45(0.05)$ & \\
\hline 3 & 5 & $5.69(0.55)$ & \\
\hline 4 & 5 & $6.88(0.26)$ & \\
\hline
\end{tabular}

*ANOVA test with significance $p<0.05$. Group 1 - untreated nanofiller composite resin Group 2 - untreated nanohybrid composite resin, Group 3 - nanofiller composite resin with treatment, Group 4 - nanohybrid composite resin with treatment

TABLE 2. Mean values, standard deviation, and significance of barium leaching (\%) in all groups

\begin{tabular}{|c|c|c|c|}
\hline Group & $n$ & Mean (SD deviation) & $p$-value \\
\hline 1 & 5 & $0.11(0.04)$ & \multirow[t]{4}{*}{$<0.000$} \\
\hline 2 & 5 & $0.26(0.12)$ & \\
\hline 3 & 5 & $0.10(0.08)$ & \\
\hline 4 & 5 & $1.54(0.63)$ & \\
\hline
\end{tabular}

served, which were $>3.5$. Color changes occur because of the presence of curcumin in a turmeric solution and tannin in a coffee solution. According to Ergün et al., the molecular size of coffee particles is greater than that of turmeric particles, and coffee is an extrinsic agent that most often causes discoloration of composite resin [24].

As shown in Table 2, there was a significant difference $(p<0.05)$ between group 1 (nanofiller composite resin) and group 2 (nanohybrid composite resin) due to the presence of camphorquinone content as a photoinitiator, which has a yellow pigment. The amount of camphorquinone in the nanohybrid composite resin was more than that in the nanofiller composite resin. Changes in color between groups 1 and 2 compared to those between groups 3 and 4 were statistically significantly different $(p<0.05)$. The color changes in groups 1 and 2 could generally be due to intrinsic factors, i.e., camphorquinone that was not activated, whereas both intrinsic and extrinsic factors could be responsible for the color changes in groups 2 and $4[1,25]$.

The maximum color change was detected in group 4, which was because of the methacrylate-based matrix content that has a high affinity for water. Furthermore, the EDX analysis of the nanohybrid composite resin showed a higher detachment of $1.54 \%$ of barium leaching. The brushing treatment caused a decrease in discoloration, which was due to the role of silica in a toothpaste, which could eliminate a portion of the pigment on the composite resin surface that was physically bonded $[2,10]$.

According to Wiilknitz [25], a toothpaste containing hydrated silica has the ability to eliminate extrinsic stains better due to its abrasive effect. However, if the extrinsic 


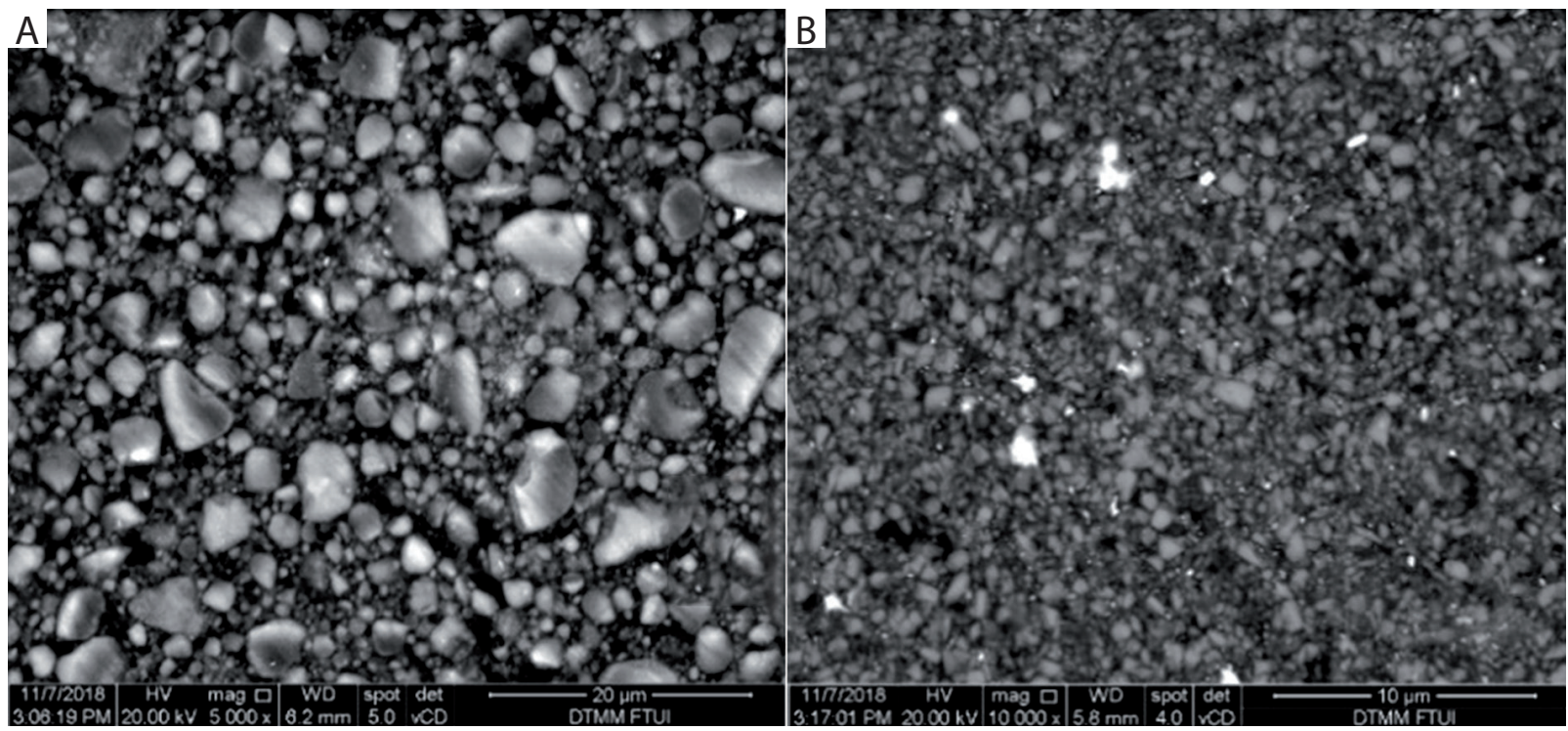

FIGURE 1. Images of surface structure and distribution of filler taken before the treatment using FESEM at a magnification of 5000x (A) surface of nanofiller composite resin, and (B) nanohybrid composite resin surface
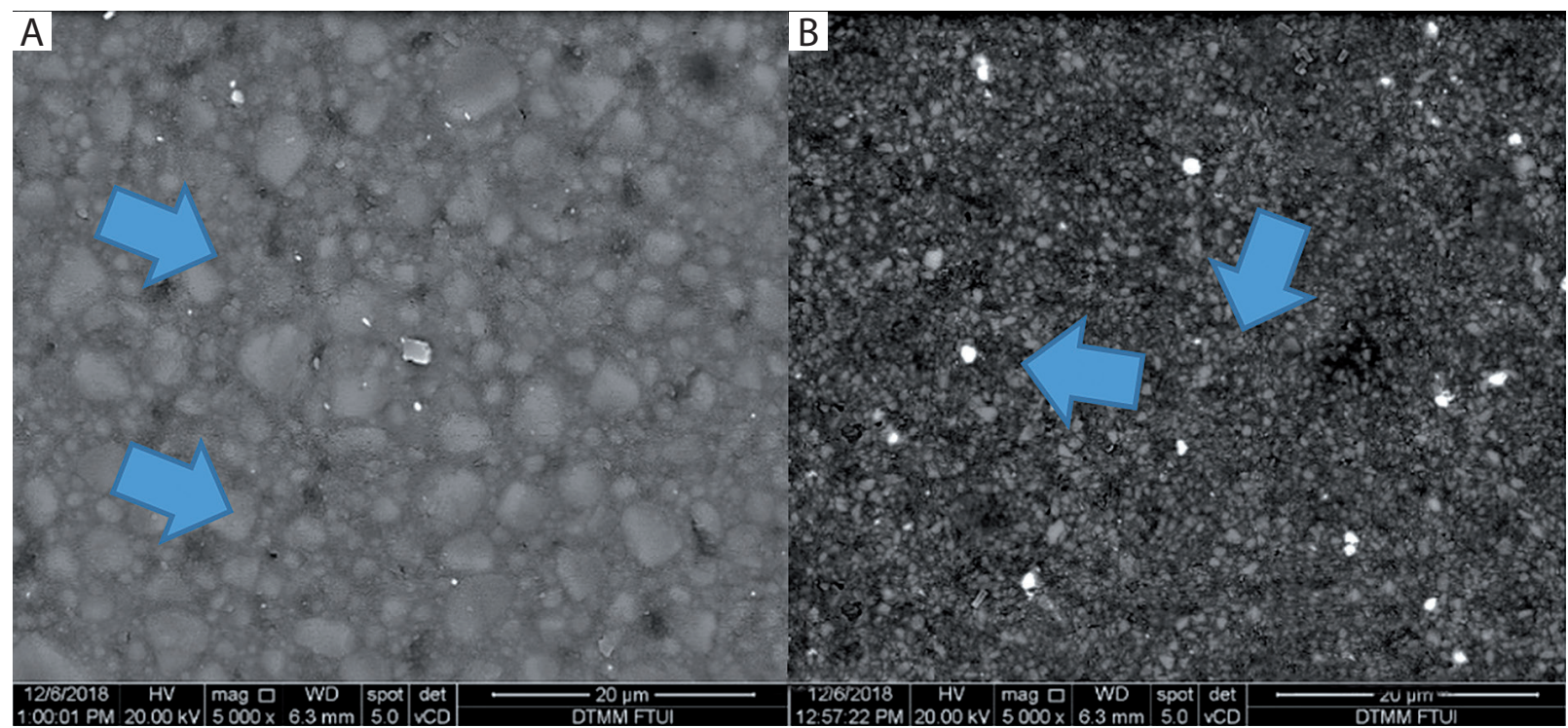

FIGURE 2. Images of surface structure after immersion in artificial saliva taken using FESEM at a magnification of $5000 \times($ A) surface structure of nanocluster-nanomeric (group 1) composite resin, and (B) nanospherical composite group surface (group 2). Blue arrows indicate the presence of several voids and fractures formed as a result of hydrolysis

pigment has a chemical bond (hydrogen), then it is difficult to remove the pigment. These results are consistent with previous studies, which have analyzed the effectiveness of a toothpaste in eliminating extrinsic stains on tooth surfaces and in composite resin restorations. However, according to Silva et al., the effect of brushing can facilitate the occurrence of color changes $[9,26,27]$.

It can be observed that the number of barium fillers decreased among the entire composite resin group, but the amount of barium leaching in group 4 was greater than that in the other groups.
In addition, there were statistically significant differences in the amount of barium leaching in the four groups $(p<0.05)$. However, according to the Bonferroni post-hoc test result, there were significant differences in group 4 compared with groups 1,2 , and $3(p<0.05)$. This was because in the process of making nanohybrid composite resins, the barium filler was mixed into the dispersant and then sprayed with a resin matrix, which was then cured. Finally, a modified filler was formed, which would then be combined with the main resin matrix of the nanohybrid composite resin, so that the surface of the nanohybrid 


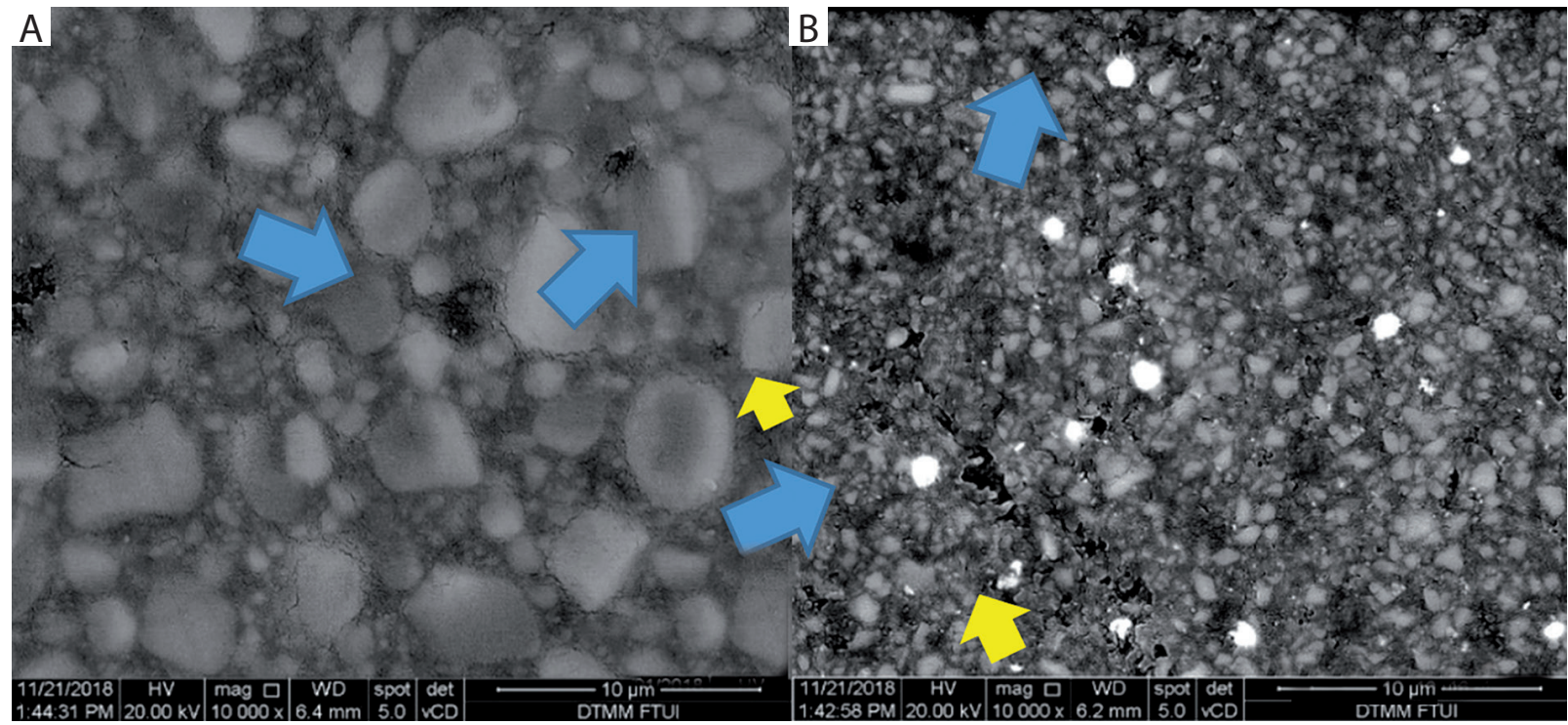

FIGURE 3. Images of surface structure after the treatment taken using FESEM at 10,000× magnification, with (A) surface structure of composite resin in group 3, and (B) surface structure of composite resin in group 4. Blue arrows indicate the voids formed due to detachment of the resin matrix and filler; yellow arrows indicate the cracks formed around the filler, which indicate that the process of hydrolysis has also occurred

composite resin specimens was very hydrophilic due to the large amount of resin matrix dressing on the surface of the composite resin [3].

In the literature, several studies have explained that barium and resin matrix fillers could be combined with a "coupling agent" known as a dispersant. The function of the merger was explained in the study of Hardinghaus et al., who aimed at deagglomerating barium fillers and preparing barium hydrophobic surfaces [28].

However, according to the study of Lin et al., dispersant-coated fillers generally reduce elastic modulus of a composite material and can slowly decrease the bond between the matrix and the filler. Meanwhile, if the filler is coated by silane to combine it with a matrix, it can strengthen a filler interaction with a matrix, so that filler release does not occur easily [29]. This was also reinforced by Chris and Roger, who explained that the use of dispersants and coupling agents (silane) was equally good, but a filler coated with a coupling agent (silane) would increase the yield strength of plastic materials; this was because silane has bonds that bind with matrices, when compared to dispersant bonds on the matrix, so that a plastic material was not easily deformed [30].

In other words, when water absorption occurs, plastic deformation is easier if the filler and the matrix are coated with a dispersant as a "coupling agent". Hydrolysis also easily occurs, because $\mathrm{H}^{+}$ions from the water directly come into contact with the methacrylate-based resin matrix in the nanohybrid composite resin, so that the barium filler can be easily released from the resin matrix $[15,31]$.

The results of this study are in accordance with the study of Soderholm et al., where they observed that the barium filler can escape from the resin matrix as time passes due to the process of hydrolysis, and is a part of the composite resin degradation. This has also been reinforced in a similar study conducted by Zhou et al., who evaluated barium leaching with immersion media in artificial saliva $[13,15]$.

However, in the nanofiller composite resins, barium fillers are coated with silane to bind them to the resin matrix. As explained in previous studies, the use of coupling agents on the surface of the filler and the matrix prevents easy occurrence of hydrolysis, which is because the use of coupling agents increases the yield strength in composites in general, so that plastic deformation does not occur as easily as described previously $[15,30]$.

In several studies, it has also been explained that the use of dispersants as coupling agents on barium filler surfaces could also be used, but this would make the barium surface hydrophobic. Hence, in the present study, the amount of barium leaching in the nanofiller composite resins was small, i.e., $0.10 \%$ and $0.11 \%$ in groups 1 and 3 , respectively. Considering the various disadvantages and advantages of dispersants and silane as mentioned earlier [28-30], it can be recommended that silane can be used as a coupling agent in a composite resin.

In addition to nanofiller composite resins, barium is present in small amounts and not as much as the number of barium fillers in nanohybrid composite resins. This can be understood by examining the surface area of the two composite resins using energy-dispersive $\mathrm{X}$-ray (EDX). In the initial examination, it was found that the average proportion of barium fillers on the surface of the nanofiller composite resin was $0.14-0.20 \% \mathrm{wt}$, whereas on the surface of the nanohybrid composite resin, it was $3.21-3.36 \%$ wt [4]. 
Another rational reason is the use of the main filler in the nanofiller composite resin consisting of non-agglomerated zirconia/silica, which adopts the concept of silica nanoparticle coating that directly binds covalently with filler zirconia $[1,2,4]$. Also, this nanosilica surface binds to silane, so that the filler in the nanofiller composite resin can bind directly to other fillers, without the need for a resin matrix as each other binder, which causes the filler in the nanofiller composite resin to not be easily released during the hydrolysis process (Figures 1-3) [1, 4].

Although nanohybrid composite resins have zirconia fillers $0.13-0.15 \%$ in weight (when characterizing surfaces with EDX). In fact, the main fillers used in the nanohybrid composite resin are barium glass and ytterbium fluoride. Barium leaching was not statistically significant $(p>0.05)$ when compared between groups 1 and 2 . This can be explained by the fact that in groups 1 and 2, only immersion in artificial saliva was conducted at $\mathrm{pH}=7$. As described by Renato et al., $\mathrm{pH}$ is also one of the causes of barium leaching. A solution with an acidic $\mathrm{pH}(\mathrm{pH}<7)$ can trigger barium leaching more easily than the artificial saliva $(\mathrm{pH}=7)$ used in this study [32].

In our study, barium leaching was not statistically significant $(p>0.05)$ when compared between groups 1 and 3. This was due to the similarity of material found in groups 1 and 3. Even though it was immersed in a solution with a different $\mathrm{pH}$, using the silane function as a coupling agent prevented the acidic $\mathrm{pH}$ from accelerating the process of hydrolysis, so that the barium filler was not easily leached [29, 30, 32].

\section{CONCLUSIONS}

Nanofiller composite resins have better color stability compared to nanohybrid composite resins and there is a strong relationship between color stability in nanofiller and nanohybrid composite resins with barium leaching.

\section{ACKNOWLEDGMENTS}

This research was supported by the directorate of Research and Community Engagement of Universitas Indonesia, funded by PITTA grant funds. We thank our colleagues from the Department of Dental Material and Metallurgy Universitas Indonesia, Prof. Dr. drg. Endang S, Sp.KG (K), drg. R. A. H. Nila Kesuma, M.PH, Sp.KG (K), drg. Bambang N, Sp.KG (K), drg. Dedy Kusuma Y, $\mathrm{M}$. Biotech, Ph. D, and our official editor.

\section{CONFLICT OF INTEREST}

The authors declare no potential conflicts of interest with respect to the research, authorship, and/or publication of this article.

\section{References}

1. Phillips WR. Science of Dental Material. $8^{\text {th }}$ ed. Philadelphia: B. Saunders Company; 2012. Available at: https://www.academia.edu/ 37341605/Philips_Science_of_Dental_Materials.pdf.

2. Craig RG, Powers JM. Restorative dental materials. $11^{\text {th }}$ ed. St. Louis: Mosby; 2002. Available at: https://www.elsevier.com/books/ dental-materials/ powers/978-0-323-31637-8.

3. Scientific Compendium Ceram. $\mathrm{x}^{\oplus}$ one vol. II. Available at: https:// www.dentsply.com/content/dam/dentsply/web/asia/Restorative/ OneResto Nav_4Seiter_2.pdf. (Accessed: December 2018).

4. Technical product profil FiltekTM Z350 XT Universal Restorative System. Available at: https://www.google.com/url?sa= $\mathrm{t} \& \mathrm{rct}=\mathrm{j} \& \mathrm{q}=\& \mathrm{esrc}=\mathrm{s} \&$ source $=$ source $=$ web \& $\mathrm{cd}=16 \& \mathrm{ved}=2 \mathrm{a}$ hUKEwjD8-DtsOLlAhVIL48KHYSRCR4QFjAPegQIABAC\&url= http\%3A\%2F\%2Fmultimedia.3m.com\%2Fmws\%2Fmedia\%2F631547O\%2Ffiltek-z350-xt-technical-product-profile.pdf\&us$\mathrm{g}=$ AOvVaw0lZ5tbX5pLIS2o54PulIXY (Accessed: December 2018).

5. Gonçalves F, Kawano Y, Pfeifer C, Stansbury JW, Braga RR. Influence of BisGMA, TEGDMA, and BisEMA contents on viscosity, conversion, and flexural strength of experimental resins and composites. Eur J Oral Sci 2009; 117: 442-446.

6. Chittem J, Sajjan GS, Kanumuri MV. Spectrophotometric evaluation of colour stability of nanohibrid composite resin in commonly used food colourants in Asian countries. J Clin Diagn Res 2017; 11: ZC61-ZC65.

7. Al Shetili MS, Al-Omari M. Color stability of nano-filled, micro-hybrid, and siloranebased dental composite resin materials. Saudi J Oral Sci 2016; 3: 42-48.

8. Lee YK, Powers JM. Discoloration of dental resin composites after immersion in a series of organic and chemical solutions. J Biomed Mater Res B Appl Biomater 2005; 73: 361-367.

9. Silva T, Sales AL, Pucci CR, Borges AB, Torres CR. The combined effect of food-simulating solutions, brushing and staining on color stability of composite resins. Acta Biomater Odontol Scand 2017; 3: 1-7.

10. Khan MK, Bokhari SA, Haleem A, et al. Extrinsic stain removal with a toothpowder: a randomized controlled trial. Int J Health Sci 2014; 8: 269-274.

11. Al-Bader RM, Ziadan KM, Al-Ajely MS. Water adsorption characteristics of new dental composites. Int J Med Res Health Sci 2015; 4: 281-286.

12. Zhou M, Drummond JL, Hanley L. Barium and strontium leaching from aged glass particle/resin matrix dental composites. Dent Mater 2005; 21: 145-155.

13. Tarumi H, Torii M, Tsuchitani Y. Relationship between particle size of barium glass filler and water sorption of light-cured composite resin. Dent Mater J 1995; 14: 37-44.

14. Söderholm KJ. Hydrolytic degradation of dental composites and effects of silane-treatment and filler fraction on compressive strength and thermal expansion of composites. 1984. Available at: https://www.diva-portal.org/smash/get/diva2:1152727/FULLTEXT03.pdf.

15. Söderholm KJ, Mukherjee R, Longmate J. Filler leachability of composites stored in distilled water or artificial saliva. J Dent Res 1996; 75: 1692-1699.

16. Asaka Y, Miyazaki M, Aboshi H, et al. EDX fluorescence analysis and SEM observations of resin composites. J Oral Sci 2004; 46: 143-148.

17. Heshmat M, Hoorizad Gangkar M, Emami Arjomand M. Colour stability of three composite resins following accelerated artificial aging. J Islamic Dent Assoc Iran 2014; 26: 90-95.

18. de Morais RC, Garcia Lda F, Cruvinel DR, Panzeri Pires-de-Souza Fde C. Color stability and surface roughness of composite submitted to different types and periods of finishing/polishing: physical properties of composites. J Contemp Dent Pract 2015; 16: 565 570.

19. Yulianto HDK. Degradation of dental resin composites during intra-oral wear. University of Groningen; 2017. Available at: https:// www.rug.nl/research/portal/files/50117858/Chapter_5.pdf. 
20. Lu H, Roeder LB, Lei LE, Powers JM. Effect of surface roughness on stain resistance of dental resin composites. J Esthet Restor Dent 2005; 17: 102-108.

21. Lee YK, Lu H, Oguri M, Powers JM. Changes in color and staining of dental composite resins after wear simulation. J Biomed Mater Res B Appl Biomater 2007; 82: 313-319.

22. Mutlu-Sagesen L, Ergün G, Ozkan Y, Semiz M. Color stability of a dental composite after immersion in various media. Dent Mater J 2005; 24: 382-390.

23. Alshali RZ, Silikas N, Satterthwaite JD. Degree of conversion of bulk-fill compared to conventional resin-composites at two time intervals. Dent Mater 2013; 29: e213-e217.

24. Seybert EK. Hydrous silica gel containing dentifrice. US Patent. US 4,153,680. 1979. Available at: https://patentimages.storage. googleapis.com/3e/5a/5e/53e7e8b3281bc3/US4303641.pdf.

25. Wilknitz P. Cleaning power and abrasivity of European toothpastes. Adv Dent Res 1997; 11: 576.

26. Hardinghaus F. Deagglomerated barium sulfate. US Patent. US 7,846,409. 2010. Available at: https://patentimages.storage. googleapis.com/d7/6e/f9/4e8ef01d062103/US7846409.pdf.

27. Lin CT, Lee SY, Keh ES, et al. Influence of silanization and filler fraction on aged dental composites. J Oral Rehab 2000; 27: 919-926.

28. DeArmitt C, Roger R. Dispersants and coupling agents. In: Applied Plastics Engineering Handbook. William Andrew Publishing; 2011, p. 501-516. Available at: https:/www.researchgate.net/ publication/236133348_Applied_Plastics_Engineering_Handbook.

29. Eliades T, Brantley WA, Watts DC. Dental material in vivo: aging and related phenomena. Eliades G (ed.). Surrey eGB GB: Quintessence; 2003. Available at: https://www.researchgate.net/publication/272741072_Dental_materials_in_vivo_ageing_and_related_phenomena.

30. Cilli R, Pereira JC, Prakki A. Properties of dental resins submitted to $\mathrm{pH}$ catalysed hydrolysis. J Dent 2012; 40: 1144-1150. 